\title{
Public
}

\section{Discovery and Structure-Based Design of Macrocyclic Peptides}

\section{Targeting STUB1}

Simon $\mathrm{Ng}, \ddagger^{\mathrm{a}}$ Alexander C. Brueckner, $\$ \S^{\mathrm{b}}$ Soheila Bahmanjah, ${ }^{\mathrm{b}}$ Qiaolin Deng, $\S^{\mathrm{b}}$ Jennifer M. Johnston, ${ }^{b}$ Lan Ge, ${ }^{c}$ Ruchia Duggal, ${ }^{d}$ Bahanu Habulihaz, ${ }^{e}$ Benjamin Barlock, ${ }^{d}$ Sookhee Ha, ${ }^{b}$ Ahmad Sadruddin, ${ }^{a}$ Constance Yeo, ${ }^{a}$ Corey Strickland, $\S^{b}$ Andrea Peier, ${ }^{\dagger}$ Brian Henry, ${ }^{a}$ Edward C. Sherer, ${ }^{\text {tb }}$ Anthony W. Partridge ${ }^{\star}{ }^{a}$

aMSD, Quantitative Biosciences, 8 Biomedical Grove, 138665, Singapore.

bMerck \& Co., Inc., Computational \& Structural Chemistry, 2000 Galloping Hill Road, Kenilworth, New Jersey 07033, United States.

'Merck \& Co., Inc., Cell Sciences Innovation, 2000 Galloping Hill Road, Kenilworth, New Jersey 07033, United States

dMerck \& Co., Inc., ADME Group 2, 33 Avenue Louis Pasteur, Boston, Massachusetts 02115, United States.

eMerck \& Co., Inc., PPDM ADME Transporters \& In Vitro Technology, 126 East Lincoln Ave, Rahway, New Jersey 07065, United States.

'Merck \& Co., Inc., Screening \& Compound Profiling, 2000 Galloping Hill Road, Kenilworth, New Jersey 07033, United States

$\$$ These authors contributed equally

*Corresponding authors: awpartridge@gmail.com, edward_sherer@merck.com

$\S$ Current affiliation:

Alexander C. Brueckner - Bristol Myers Squibb, 3551 Lawrenceville Rd, Lawrence Township, NJ 08648, United States

Corey Strickland and Qiaolin Deng - Proteovant Therapeutics, 2500 Renaissance Boulevard, King of Prussia, PA 19406, United States

Anthony W. Partridge - Roivant Sciences, 451 D St, Boston, MA 02210, United States 


\section{Public}

\section{Abstract}

STIP1 homology and U-Box containing protein 1 (STUB1) plays a key role in maintaining cell health during stress and aging. Recent evidence suggested STUB1 also helps regulate immunity with the potential of clearing malignant cells. Indeed, we and others have shown that STUB1 is a pivotal negative regulator of interferon gamma sensing - a process critical to the immunosurveillance of tumors and pathogens. Thus far, investigation of STUB1's role relies mostly on genetic approaches as pharmacological inhibitors of this protein are lacking. Identification of a STUB1 tool compound is important as it would allow therapeutically relevant target validation in a broader sense. Accordingly, we leveraged phage display and computational modeling to identify and refine STUB1 binders. Screening of $>10^{9}$ macrocyclic peptides resulted in several conserved motifs as well as structurally diverse leads. Co-crystal structure of the peptide hit and STUB1 has enabled us to employ structure-based in silico design for further optimization. Of the modifications employed, replacing the hydrophilic solvent-exposed region of the macrocyclic peptides with a hydrophobic scaffold improved cellular permeability, while the binding conformation was maintained. Further substitution of the permeability-limiting terminal aspartic acid with a tetrazole bioisostere retained the binding to certain extent while improving permeability, suggesting a path forward. The current lead, although not optimal for cellular study, provides a valuable template for further development into selective tool compounds for STUB1 to enable target validation. 


\section{Public}

\section{Introduction}

STIP1 homology and U-Box containing protein 1 (STUB1), also known as the carboxy-terminus of Hsp70-interacting protein (CHIP), is central for the homeostasis of cellular proteins ${ }^{1-2}$ Canonically, STUB1 E3 ligase interacts with chaperone proteins such as Hsp70 and Hsp90 during the refolding cycle. ${ }^{3}$ Proteins that cannot be rescued are instead polyubiquitylated by STUB1 and are thus directed for disposal through autophagy, the endocytic-lysosomal pathway and/or the ubiquitin-proteasomal pathway. The list of protein substrates targeted by STUB1 is ever expanding, ${ }^{4-5}$ although controversies regarding some of the targets remain. ${ }^{6}$ Generally, most studies agreed that STUB1 is a guardian of cells during stress and aging. Indeed, deficiency of STUB1 in mice ${ }^{7-8}$ and worms ${ }^{9}$ accelerates the aging process. These findings are further corroborated in humans where patients harboring loss-of-function mutation in STUB1 exhibited aging phenotypes. ${ }^{10}$ More recently, evidence suggests that STUB1 might play additional roles in regulating immunity. ${ }^{11-14}$ In accordance, we and others have shown that STUB1 constitutively constrains interferon gamma sensing by downregulating the interferon gamma cell surface receptor. ${ }^{15-17}$ As a result, inactivation of STUB1 inhibited growth of cancer cell lines in vitro after prolonged treatment with interferon gamma, ${ }^{17}$ and can facilitate clearance of STUB1-deficient cells by cytotoxic T cells in a mouse model. ${ }^{16}$ However, these studies exclusively deleted STUB1 in malignant cells while leaving the gene unperturbed in immune and stromal cells. Since inhibition of STUB1 in these latter tissues may influence therapeutic effect and on-target toxicities, finding a tool compound for STUB1 will pave the way for chemical genetics. Such approach will enable the 


\section{Public}

validation of the target and its putative functions in a systemic and reversible manner that is difficult to achieve using genetic inactivation.

Recent efforts using a synthetic combinatorial library identified a linear peptide (LWWPD) as a potent binder for STUB1. ${ }^{18}$ As linear peptides usually lack cellular permeability, ${ }^{19}$ this sequence was fused to an arginine-rich sequence to overcome this limitation..$^{20}$ However, peptides relying on poly-arginine tags for cellular entry can induce mast cell degranulation, ${ }^{21-22}$ a phenomenon confirmed in our recent work of targeting KRas with arginine-rich peptides. ${ }^{23}$ This potentially fatal condition could make such constructs challenging to apply in vivo. As an alternative approach, macrocyclic peptides represent a promising modality for in vivo tool compound development with many desirable attributes including improved stability, cellular permeability, oral bioavailability, and the potential to target shallow or flat protein surfaces,,$^{23-27}$ configurations that are typical for E3 ligases.

Herein, we disclose a novel class of macrocyclic peptides targeting STUB1. The discovery capitalized on three enabling technologies: (i) chemical post-translational cyclization of $>10^{9}$ phage-encoded peptide libraries; (ii) deep sequencing for hit identification; and (iii) computational design. In this study, a new computational approach was established to design and refine the macrocyclic peptides with improved cellular permeability while maintaining the active binding conformation. 


\section{Public}

\section{Results and discussion}

\section{Hit Identification with Phage Display}

To initiate the screening campaign, we generated three phage libraries, namely Lib1, Lib2 and Lib3, each with $>10^{9}$ experimental diversity (Table S1). The libraries contain 4 or 6 residues of any 20 amino acids scrambled with 2 or 4 cysteine residues (Fig. 1a and Fig. S1a). The four-cysteines strategy, inspired by Kale et al., ${ }^{28}$ allowed us to sample vast structural diversity and various macrocyclic sizes through pairing of the cysteines (Fig. S1a). ${ }^{28}$ Although spontaneous cyclization through disulfide bond is a facile approach, its susceptibility to reduction is a liability for developing intracellularly active peptides. ${ }^{23}$ To mitigate this issue, we sought to replace the disulfide bond with a nonreducible linkage prior to the screen. ${ }^{28-30}$ First, we performed post-translational modification of all three phage libraries with either 1,3-dibromoacetone (Fig. 1b) or $\alpha, \alpha^{\prime}-$ dibromo-p-xylene (Fig. S1b). Next, the modified libraries were screened against STUB1 (Fig. 1c and Fig. S1c). To account for non-specific binding events, an irrelevant E3 ligase (SPOP) was introduced as a negative control during the screen. We recovered the bound phages and decoded the phage DNAs using Illumina NextSeq after a single round of selection. Through differential enrichment analysis,, ${ }^{31-33}$ we identified 63 peptide sequences from 1,3-dibromoacetone-modified Lib1. These sequences were selectively enriched for STUB1, but not for SPOP (Fig. 1c). The enriched peptides could be clustered into four families based on their cysteines position (Fig. 1d). Eventually, we identified several closely related consensus motifs across the sequence families, suggesting a robust screen (Fig. 1d). Similarly, screening of bicyclic peptides, encoded by Lib2 and 


\section{Public}

Lib3, resulted in highly conserved motifs, suggesting consistent and reproducible discovery (Fig. S1c). We shortlisted three major families for synthesis - two from 1,3dibromoacetone-modified Lib1 and one from $\alpha, \alpha^{\prime}$-dibromo-p-xylene-modified Lib2. To validate their binding to STUB1, we used thermal shift assay (TSA) and isothermal titration calorimetry (ITC) - which are orthogonal and indispensable in the hit validation funnel since each approach has its own merits and limitations. ${ }^{34-35}$ Terminal Ala and Gly were included in the sequence (Table 1), in order to match how the peptides were originally displayed on the phage (Fig. 1b). Unlike Lib1, the peptides enriched from Lib2 could potentially form three regioisomers, which were synthesized individually for binding validation. Indeed, STUB1 could bind to all five synthesized peptides (1-5) with submicromolar affinity (Table 1). Among them, the bicyclic peptide 5 exhibited the best binding $\left(\mathrm{K}_{\mathrm{D}}=20-40 \mathrm{nM}, \Delta \mathrm{T}_{\mathrm{m}}=+11.0^{\circ} \mathrm{C}\right)$. To strike a balance between the binding affinity and cellular permeability, we took the size of the peptides into account. ${ }^{36-37}$ We prioritized peptide 1 for further optimization, after considering its much smaller size (1091 Da) and simpler structure relative to peptide 5 (1475 Da). 


\section{Public}



Fig. 1 Screening of 1,3-dibromoacetone-modified Lib1 against SPOP and STUB1. (a) The design and format of Lib1 displayed on M13 phage. X denotes any 20 amino acids encoded by NNK codon. (b) TCEP reduction and subsequent alkylation of the Cys thiols yielded a ketone-bridged macrocyclic peptide library. (c) Selection of the modified Lib1 against immobilized SPOP and STUB1, followed by deep sequencing and differential enrichment analysis, identified 63 peptides binding exclusively to STUB1. Each row and column of the heatmap represents a unique sequence and an independent panning experiment, respectively. (d) Clustering of 63 enriched peptides revealed four sequence families and their consensus motif. Asterisks next to the sequence number highlight the families shortlisted for the synthesis of peptide $\mathbf{1}$ and $\mathbf{2}$.

Table 1. TSA and ITC validation of macrocyclic peptides shortlisted from phage display.

\begin{tabular}{|c|c|c|c|c|c|}
\hline Peptide & Sequence $^{a}$ & Linkage & MW & $\Delta \mathrm{T}_{\mathrm{m}}$ & \\
\hline & & between Cys & $(\mathrm{Da})$ & $\left({ }^{\circ} \mathrm{C}, \mathrm{TSA}\right)^{b}$ & $(\mathrm{nM}, \mathrm{ITC})$ \\
\hline 1 & $\mathrm{NH}_{2}-\mathrm{ACSS}$ IWCPDG$-\mathrm{CONH}_{2}$ & $\mathrm{CH}_{2} \mathrm{C}=\mathrm{OCH}_{2}$ & 1091 & +9.9 & 160 \\
\hline 2 & $\mathrm{NH}_{2}-\mathrm{ACS} I W N P Y C G-\mathrm{CONH}_{2}$ & $\mathrm{CH}_{2} \mathrm{C}=\mathrm{OCH}_{2}$ & 1166 & +8.7 & 150 \\
\hline 3 & $\mathrm{NH}_{2}-\mathrm{ACS}$ IWCPDCCNG-CONH & $p$-xylyl & 1475 & +4.5 & 3200 \\
\hline 4 & $\mathrm{NH}_{2}-\mathrm{ACS}$ IWCPDCCNG-CONH & $p$-xylyl & 1475 & +8.7 & 77 \\
\hline 5 & $\mathrm{NH}_{2}-\mathrm{ACS}$ IWCPDCCNG-CONH & p-xylyl & 1475 & +11.0 & $20 ; 40^{c}$ \\
\hline
\end{tabular}

${ }^{a}$ Each pair of cysteine crosslink is highlighted in different colors. ${ }^{b}$ Shift in melting temperature $\left(\Delta T_{\mathrm{m}}\right)$ of STUB1 in the presence of peptide relative to DMSO control. $\Delta T_{\mathrm{m}}$ is derived from technical duplicates. Complete datasets are available in supplementary information. ${ }^{C} K_{D}$ values from two independent experiments were shown explicitly. 


\section{Public}

Structure-activity relationship of peptide 1

To explore the structure-activity relationship (SAR) of peptide 1, we removed the terminal residues (Ala and Gly) and observed better binding of 6 relative to 1 . Furthermore, substituting the C-terminal amide of Asp with an acid (7) greatly enhanced its binding relative to 6 (Table 2). In contrast, the affinity was substantially reduced upon deletion of the terminal Asp (compare 9 and 10), highlighting its importance for binding. These observations support earlier findings that the C-terminal Asp of Hsp70/Hsp90 is a key residue governing the interactions between chaperones and STUB1 during their complex formation. ${ }^{38-39}$ Unlike the inflexibility of the C-terminus, modifications are welltolerated at the $\mathrm{N}$-terminus. Adding 6-azido-L-lysine (AzK) (8) or substituting Ser with AzK (9), at a position close to the $\mathrm{N}$-terminus, did not affect their binding affinity as compared to 7. The freedom to incorporate AzK allowed us to introduce a bioorthogonal reactive handle for NanoClick ${ }^{40}$ - a cellular assay for measuring peptide permeability (vide infra).

Table 2. Structure-activity relationship of peptide 1.

\begin{tabular}{lrlll}
\hline Peptide & Sequence $^{a}$ & $\begin{array}{l}\text { Linkage } \\
\text { between Cys }\end{array}$ & $\begin{array}{l}\Delta \mathrm{Tm}_{\mathrm{m}} \\
\left({ }^{\circ} \mathrm{C}, \mathrm{TSA}\right)^{b}\end{array}$ & $\begin{array}{l}\mathrm{K}_{\mathrm{D}} \\
(\mathrm{nM}, \mathrm{ITC})\end{array}$ \\
\hline $\mathbf{6}$ & $\mathrm{Ac}-\mathrm{CSSIWCPD}-\mathrm{CONH}{ }_{2}$ & $\mathrm{CH}_{2} \mathrm{C}=\mathrm{OCH}_{2}$ & +13.4 & 30 \\
$\mathbf{7}$ & $\mathrm{Ac}-\mathrm{CSSIWCPD}-\mathrm{COOH}$ & $\mathrm{CH}_{2} \mathrm{C}=\mathrm{OCH}_{2}$ & +21.3 & $2.3 ; 15^{c}$ \\
$\mathbf{8}$ & $\mathrm{Ac}-\mathrm{C}-\mathrm{AzK}-\mathrm{SIWCPD}-\mathrm{COOH}$ & $\mathrm{CH}_{2} \mathrm{C}=\mathrm{OCH}_{2}$ & +21.7 & $4.1 ; 49^{c}$ \\
$\mathbf{9}$ & $\mathrm{Ac}-\mathrm{AzK}-\mathrm{CSSIWCPD}-\mathrm{COOH}$ & $\mathrm{CH}_{2} \mathrm{C}=\mathrm{OCH}_{2}$ & +21.8 & 25 \\
$\mathbf{1 0}$ & $\mathrm{Ac}-\mathrm{AzK}-\mathrm{CSSIWCP}-\mathrm{CONH} 2$ & $\mathrm{CH}_{2} \mathrm{C}=\mathrm{OCH}_{2}$ & +4.5 & $1070 ; 1870^{c}$ \\
$\mathbf{1 1}$ & $\mathrm{Ac}-\mathrm{AzK}-\mathrm{CSSIWCPD}-\mathrm{CONH}_{2}$ & $\mathrm{CH}_{2} \mathrm{C}=\mathrm{OCH}_{2}$ & +14.8 & $3.7 ; 21^{c}$ \\
$\mathbf{1 2}$ & $\mathrm{Ac}-\mathrm{AzK}-\mathrm{CSSIWCPD}-\mathrm{CONH}_{2}$ & Disulfide & +10.5 & $52 ; 49^{c}$
\end{tabular}




\section{Public}

13

AC-AzK-SSSIWSPD-CONH $2 \quad \mathrm{NA}^{d}$

$+9.8$

$343 ; 326^{c}$

${ }^{a} \mathrm{Ac}$ is acetyl. AzK is 6-azido-L-lysine. ${ }^{b}$ Shift in melting temperature of STUB1 in the presence of peptide relative to DMSO control. $\Delta \mathrm{T}_{\mathrm{m}}$ is derived from technical duplicates. Complete datasets are available in supplementary information. ${ }^{c} K_{D}$ values from two independent experiments were shown explicitly. ${ }^{d}$ Not applicable (NA).

Cyclization often enhances the affinity of linear peptides, owing to reduced conformational freedom and a smaller entropic penalty paid upon target binding. However, rational design of the cyclic linkage and its point of connection is not a straightforward task - subtle changes can substantially perturb the conformation of macrocyclic peptides, thereby affecting binding. Here, we sought to test these hypotheses by exploring different linkages (Fig. 2a). First, changing the ketone bridge (11, $\mathrm{K}_{\mathrm{D}}=3.7-21$ $n M)$ into a disulfide linkage $\left(12, K_{D}=49-52 n M\right)$ resulted in 4-fold lower binding affinity (Fig. 2 and Table 2). Second, a linear version of 11, where the two Cys were replaced with cyclization-incompetent Ser $\left(\mathbf{1 3}, \mathrm{K}_{\mathrm{D}}=\mathbf{3 2 6}-343 \mathrm{nM}\right)$, exhibited 27-fold lower affinity for STUB1 (Fig. 2 and Table 2). As expected, the ketone linkage was optimal for binding, whereas a more compact (disulfide) or a completely unconstrained (linear) peptide were inferior. Together, these findings substantiate our efforts of modifying the phagedisplayed peptide libraries prior to screening and validate the advantages offered by cyclization. 


\section{Public}
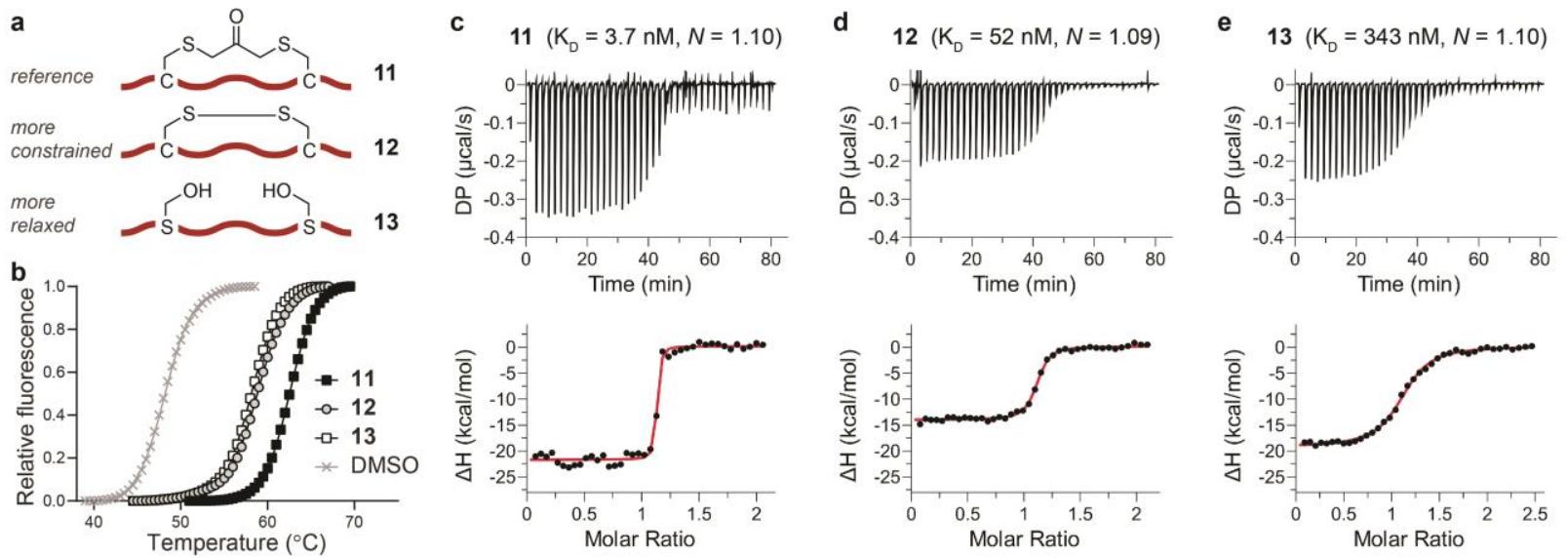

Fig. 2 The effect of peptide cyclization for binding to STUB1 (aa25-aa153). (a) Figure showing the chemical structure of crosslink in peptide 11-13. (b) Melting curves of STUB1 $(10 \mu \mathrm{M})$ in the presence of the peptides $(100 \mu \mathrm{M})$. For ease of comparison, the maximal and minimal fluorescence intensity in each data set were normalized to 1.0 and 0.0 respectively. Mean from technical duplicates were plotted. The s.d. is too small to be visible. (c-e) ITC titrations of the peptides $(200 \mu \mathrm{M})$ into STUB1 $(20 \mu \mathrm{M})$ at $25^{\circ} \mathrm{C}$. The dissociation constant $\left(K_{D}\right)$ and binding stoichiometry $(M)$ were derived from the curve fitting (red curve). The lack of inflection points in the titration curve of 11, due to its high binding affinity, resulted in an imprecise estimate of $\mathrm{K}_{\mathrm{D}}$. Complete datasets of repeat experiments are available in supplementary information.

\section{$\underline{\text { In silico design and structure-based shrinkage of macrocycle }}$}

We obtained a crystal structure of peptide 1 bound to the tetratricopeptide repeat (TPR) region of STUB1 (Fig. 3a). Mapping the protein-ligand interactions in the crystal structure revealed that the crosslink between $\mathrm{C} 2 / \mathrm{C} 7$ and the $\mathrm{N}$-terminal residues $\mathrm{A} 1, \mathrm{C} 2$, and S3 were solvent-exposed and did not interact with the protein. To obtain potent and permeable peptides, five modified series were proposed and evaluated computationally (Table S2). Series 1 and 2 - replacement of the hydrophilic solvent-exposed region (Fig. 3) and neutralization of the acidic sidechain of D9 (Fig. 4), respectively - will be discussed in this manuscript. Series 3-5 were ultimately deprioritized based on modeling results and will not be discussed. 


\section{Public}
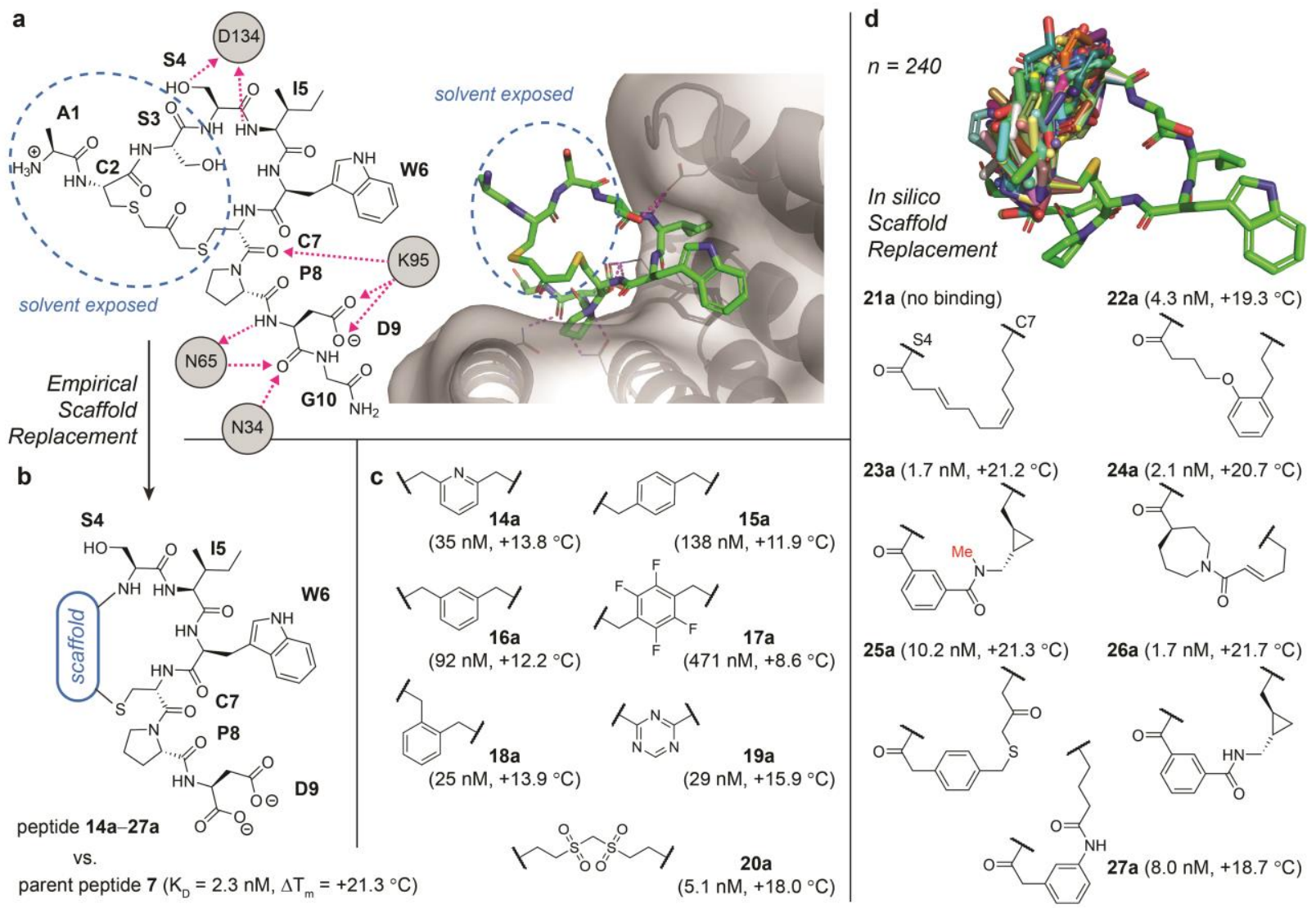

Fig. 3 Empirical and in silico replacement of solvent-exposed region with various scaffolds. (a) Crystal structure and interaction map for peptide 1 bound to STUB1. Peptide 1 is shown as green sticks, the TPR region of STUB1 as grey cartoon/surface/lines, and protein-ligand interactions as magenta lines. The solvent-exposed region is emphasized with a blue dotted oval. (b, c) Initial efforts to replace the solvent-exposed region with scaffolds based on previous work. ${ }^{41}$ (d) Scaffold replacement for the same region by in silico screening of 240 potential linkers. The 3D representations are arbitrary minimized conformations. Peptide 7 was the parent peptide for scaffold replacement $(b-d)$. The structure of scaffold for each synthesized peptide and the representative $K_{D}$ and $\Delta T_{m}$ (in parentheses) were shown explicitly (c, d). Complete datasets and repeat experiments are available in supplementary information (c, d).

Optimization was ultimately applied to peptide 7 (Fig. 3b and Table 2), a close analog of peptide $\mathbf{1}$. Both peptide $\mathbf{1}$ and peptide $\mathbf{7}$ are identical in cyclic structure, but the terminal $\mathrm{A} 1$ and $\mathrm{G} 10$ residues were removed in peptide 7 and a carboxylic acid was 


\section{Public}

exposed at the C-terminus. For clarity, the residues in peptide 7 are labeled in the same way as peptide 1 (i.e., the macrocycle is formed from $\mathrm{C} 2$ to $\mathrm{C} 7$ in both peptides).

Initial efforts aimed at replacing the solvent-exposed region using scaffolds inspired by the works of Kale et al. (Fig. 3b and 3c). ${ }^{41}$ The scaffolds were not only commercially available but were structurally diverse, allowing us to quickly sample a wide variety of macrocyclic backbones and conformations. Overall, this empirical approach generated peptides (Fig. 3c, 14a-19a) with >10-fold lower binding affinity for STUB1 than the parent peptide 7 (Fig. 3b). Interestingly, peptide 20a $\left(\mathrm{K}_{\mathrm{D}}=5.1 \mathrm{nM}, \Delta \mathrm{T}_{\mathrm{m}}=+18.0^{\circ} \mathrm{C}\right)$ bound to STUB1 with an affinity comparable to peptide $7\left(\mathrm{~K}_{\mathrm{D}}=2.3 \mathrm{nM}, \Delta \mathrm{T}_{\mathrm{m}}=+21.3^{\circ} \mathrm{C}\right)$ - an exception when a more flexible scaffold was used.

Next, we employed in silico ligand design to guide the replacement of solventexposed regions in peptide 7 using novel hydrophobic scaffolds. To strike a balance between cellular permeability and binding affinity, we established a multi-parameter optimization (MPO) to account for bioactive conformer retention, molecular weight, and AlogP (Fig. S2 and Table S3). To maintain the binding affinity, a retention of the bioactive conformation is required. It is well understood that modifications to the chemical structure can have drastic effects on molecular shape ${ }^{42}$ Each proposed modification was subjected to a Replica Exchange with Solute Tempering (REST2) simulation in a water cube (see supplementary information) to ensure the bioactive conformation was retained. ${ }^{43}$ REST2 is well suited for sampling the complex conformational landscape of macrocyclic peptides due to the ability to overcome kinetic trapping between local energy minima by simulating and swapping solutes between various temperature conditions. We can assume that if the bioactive conformation occurs frequently enough in solvent, it will be accessible and 


\section{Public}

available for binding to STUB1. When comparing each timestep of the simulation for peptide 1 to the crystal structure, it was noted that the bioactive conformation was visited $0.5 \%$ of the simulation time. Therefore, each proposed modification was assumed to retain the bioactive conformation if the sampling percentage was at or above $0.5 \%$ (Fig. S2).

The permeability of a given compound can be approximated using the partition coefficient (AlogP), the diffusion coefficient (molecular weight dependent), and the width of the cell membrane. ${ }^{44-45}$ In this case, the width of the cellular membrane is assumed to be the same for all peptides. Therefore, AlogP and molecular weight are the main driving forces for permeability. Based on previous reports for the successful design of permeable peptides $^{36-37}$ and in-house experience, a molecular weight less than $1000 \mathrm{Da}$ and AlogP values between 1 and 6 were deemed desirable (Fig. S2).

Eventually, 240 peptides were proposed by Scaffold Replacement in MOE (Fig. 3d and Table S2). ${ }^{46}$ Prioritization based on synthetic feasibility offered 15 designs, of which seven (Fig. 3d, 21a-27a) were shortlisted by MPO analysis as described above (Fig. S2 and Table S3). After synthesis, all peptides (22a-27a) proposed by the computational design, except for 21a, bound to STUB1 with $\mathrm{K}_{\mathrm{D}}$ and $\Delta \mathrm{T}_{\mathrm{m}}$ ranging 1.7-10.2 $\mathrm{nM}$ and 18.7-21. $7^{\circ} \mathrm{C}$, respectively. Among these six peptides (22a-27a), two are slightly inferior (22a and 27a) while the rest are on-par with their parent peptide $7\left(K_{D}=2.3 n M\right.$ and $\Delta T_{m}$ $\left.=21.3^{\circ} \mathrm{C}\right)$. Nonetheless, lack of binding of 21a was unexpected. Further investigation of 21a showed that, during the synthesis of its linker, an $\alpha, \beta$-unsaturated isomer was observed in addition to the desired $\beta, \gamma$-unsaturated isomer. The observed loss of binding affinity in $\mathbf{2 1 a}$ is likely due to scrambling of the peptide linker over time. Overall, in silico 


\section{Public}

approaches achieved a greater probability of success (six out of seven, Fig. 3d), relative to the empirical approach (one out of seven, Fig. 3c), in shrinking the macrocyclic peptide while maintaining its bioactive conformation.

\section{Cellular permeability of macrocyclic peptides}

The success of replacing the hydrophilic solvent-exposed regions with hydrophobic scaffolds prompted us to evaluate whether this strategy could improve peptide permeability. Accordingly, we employed NanoClick, a target-agnostic assay developed previously, to measure the cellular uptake of molecules via different mechanisms, including but not limited to endocytosis, membrane translocation, and passive permeability. ${ }^{40}$ This assay quantifies an azide-containing test compound, e.g., peptide, such that it will pre-compete with a permeable azide-containing dye molecule which covalently labels an intracellular protein sensor in HeLa cells. Accordingly, test compounds with higher permeability in NanoClick will exhibit smaller $\mathrm{EC}_{50}$ value in competing off the permeable dye molecule.

We emphasized that D9 residue of the peptides (Fig. 3b, 18a-27a) is crucial for binding but deleterious for permeability. To truly measure the impact of scaffolds on peptide permeability and to rule out any complexity caused by target binding, we synthesized a new set of peptides (18b-27b) based on 18a-27a (Fig. 3b) by deleting the D9 and substituting the S4 with an azido-Lys (AzK). The same treatments were applied to peptide $\mathbf{7}$ to afford $\mathbf{7 b}$ as an appropriate reference peptide. These Asp-deleted, azidecontaining peptides (7b and 18b-27b, see Fig. 3c and 3d for scaffold structure) were evaluated for their cellular uptake using the NanoClick assay along with a permeable $\alpha-$ 


\section{Public}

helical stapled peptide (azido-ATSP-7041) - an analog of an advanced p53/MDM2 clinical candidate that we had applied extensively as a benchmark for permeability. ${ }^{40,47-}$ ${ }^{48}$ Among the peptides tested (7b and $\mathbf{1 8 b} \mathbf{- 2 7} \mathbf{b}$, Table 3 ), the top five most permeable peptides are, in descending order: $\mathbf{2 2 b}, \mathbf{1 8 b}, \mathbf{2 1 b}, \mathbf{2 5 b}$, and $\mathbf{2 3 b}$. Of these five, the permeability of 22b nearly matched azido-ATSP-7041, at an 18-hour assay time point $\left(\mathrm{EC}_{50}=220\right.$ and $136 \mathrm{nM}$, respectively), but appeared less permeable at a 4-hour time point $\left(\mathrm{EC}_{50}=1,722\right.$ and $\left.281 \mathrm{nM}\right)$. Additionally, we did not detect any passive permeation of 22b and 23b using a PAMPA assay. These findings suggest that cellular uptake of the peptides is kinetically slow and is likely less dependent on passive diffusion. We evaluated the proteolytic stability of the peptides using HeLa cell homogenates and determined half-lifes ( $\left.t_{1 / 2}\right)$ up to an upper boundary of 373 min (Table 3 ). Accordingly, 22b is unstable with a short half-life of 26 min in the cell homogenate, whereas its parent analog 22a is more stable ( $\left.t_{1 / 2}>373 \mathrm{~min}\right)$. We deduced that either the azido group and/or the C-terminal Pro in 21b-27b could be the metabolic soft spot. Indeed, the peptide became more stable (21a-27a, Table 3) after the C-terminal Pro was capped with Asp.

Table 3. Cellular stability and permeability of macrocyclic peptides.

\begin{tabular}{llll||ll}
\hline Peptide & $\begin{array}{l}\text { 4-hr. NanoClick } \\
\mathrm{EC}_{50}(\mathrm{nM})^{a}\end{array}$ & $\begin{array}{l}\text { 18-hr. NanoClick } \\
\mathrm{EC}_{50}(\mathrm{nM})^{a}\end{array}$ & $\begin{array}{l}\mathrm{HeLa}_{1 / 2} \\
(\mathrm{~min})\end{array}$ & Peptide & $\begin{array}{l}\mathrm{HeLa} \mathrm{t}_{1 / 2} \\
(\mathrm{~min})\end{array}$ \\
\hline 7b & $>10,000$ & $>10,000$ & 274 & & \\
18b & $1,566 \pm 968$ & $334 \pm 8$ & $\mathrm{ND}^{b}$ & $\mathbf{1 8 a}$ & $\mathrm{ND}$ \\
$\mathbf{1 9 b}$ & $>10,000$ & $1,960 \pm 1,044$ & 39 & $\mathbf{1 9 a}$ & $\mathrm{ND}$ \\
$\mathbf{2 0 b}$ & $>10,000$ & $2,085 \pm 1,360$ & $\mathrm{ND}$ & $\mathbf{2 0 a}$ & $\mathrm{ND}$ \\
21b & $5,225 \pm 632$ & $649 \pm 35$ & 61 & $\mathbf{2 1 a}$ & $>373$
\end{tabular}




\section{Public}

\begin{tabular}{llll||ll} 
22b & $1,722 \pm 486$ & $220 \pm 44$ & 26 & $\mathbf{2 2 a}$ & $>373$ \\
23b & $2,187 \pm 605$ & $804 \pm 108$ & 244 & $\mathbf{2 3 a}$ & ND \\
24b & $>10,000$ & $2,214 \pm 465$ & $>373$ & $\mathbf{2 4 a}$ & $>373$ \\
$\mathbf{2 5 b}$ & $1,261 \pm 207$ & $680 \pm 1$ & 137 & $\mathbf{2 5 a}$ & 204 \\
$\mathbf{2 6 b}$ & $>10,000$ & $2,111 \pm 378$ & $>373$ & $\mathbf{2 6 a}$ & $>373$ \\
$\mathbf{2 7 b}$ & $>10,000$ & $1,526 \pm 730$ & 98 & $\mathbf{2 7 a}$ & $>373$ \\
$\mathbf{2 2 c}$ & $2,380 \pm 960$ & $478 \pm 29$ & $>373$ & $\mathbf{2 2 d}$ & 213 \\
$\mathbf{2 8}$ & $>10,000$ & $>10,000$ & $\mathrm{ND}$ & & \\
$\mathbf{2 9}$ & $>10,000$ & $>10,000$ & $\mathrm{ND}$ & & \\
$\mathbf{3 0}$ & $>10,000$ & $4,106 \pm 1,136$ & $\mathrm{ND}$ & & \\
azido- & $281 \pm 91$ & $136 \pm 22$ & $>373$ & & \\
ATSP-7041 & & & & \\
\hline
\end{tabular}

${ }^{a}$ Mean \pm SD from two independent experiments were shown. For azido-ATSP-7041, data were derived from 36 independent experiments. ${ }^{b}$ Not determined (ND) due to insufficient material. " Peptides denoted with "a" and "d" were not applicable for NanoClick as these peptides do not contain an azido group. ${ }^{d}$ Azido-ATSP-7041 is a permeable peptide serving as the positive control and benchmark for the assay.

\section{Mutagenesis of Terminal Aspartic Acid}

Negatively charged molecules are deleterious for cellular permeability. ${ }^{49}$ As such, the aspartic acid of peptide 1 (indicated as D9 in Fig. 3a) was mutated in silico to neutralize the acidic sidechain (Fig. 4a). The interaction map fundamental for ligand design revealed that the D9 sidechain interacts with the sidechain of K95 in STUB1 to form a salt bridge (Fig. 3a). Therefore, mutations at this position would pose a challenge for retaining binding affinity. Nevertheless, nine designs were explored, of which three (28-30, Fig. 4b) were prioritized based on MPO analysis (Fig. S2 and Table S3). All three selected mutations have hydrogen bonding groups able to form favorable interactions with $\mathrm{K} 95$ in the hopes of mitigating the potential loss of salt bridge formation. 


\section{Public}

Sidechain mutagenesis was less successful relative to the scaffold replacement. We observed $>10$-fold lower binding affinity by mutating the acidic sidechain of peptide 6 into asparagine and histidine (28 and 29, Fig. 4c). Mutation of D9 sidechain into a tetrazole retained the binding affinity to certain extent (30, Fig. 4c). Moreover, the permeability of peptide $\mathbf{3 0}$, relative to that of $\mathbf{2 8}$ and $\mathbf{2 9}$, was marginally improved at the 18-hour time point in NanoClick assay (Table 3). These key findings suggest that substitution of the Asp sidechain with a tetrazole may offer an attractive opportunity to further fine-tune the notoriously challenging balance between binding and permeability. We noted that peptide 6 is an appropriate binding control in this study. Besides the mutated sidechain, the only difference between $\mathbf{6}$ and $\mathbf{2 8 - 3 0}$ is a substitution of S3 to AzK near the N-terminus (Fig. 4b), where these manipulations were well-tolerated as exemplified by peptides 7-9 (Table 2, also see the solvent-exposed region in Fig. 3a).

To better understand the role of C-terminal groups and the azido label on permeability and binding affinity, a new peptide 22c was synthesized (Fig. 4d). We effectively combined the best scaffold (in term of permeability) and the best D9 mutation (in term of both binding and permeability) into one peptide (22c) where a tetrazolecontaining amino acid is additionally installed at the C-terminus of peptide $\mathbf{2 2} \mathbf{b}$. Gratifyingly, the cellular uptake of $\mathbf{2 2 c}$ was only slightly worse than $\mathbf{2 2 b}$ (18-hour EC $50=$ 478 and $220 \mathrm{nM}$, respectively, Table 3), while its stability in cell homogenate was markedly improved $\left(t_{1 / 2}=26\right.$ min and $>373$ min for $\mathbf{2 2 b}$ and $22 c$ respectively, Table 3$)$. However, weak binding $\left(K_{D}>10,000 \mathrm{nM}\right)$ was observed for 22c despite the additional contribution of the tetrazole moiety. This observation indicates that the azido label at the S4 position (see peptide $\mathbf{3 0}$ in Fig. 4 b) has a detrimental effect on binding affinity of 22c 


\section{Public}

(Fig. 4d). Returning to the crystal structure and interaction map used as the foundation for ligand design (Fig. 3a), we observed that the S4 hydroxyl group interacts with the D134 residue of STUB1. After replacing the hydrogen bond donor group of S4 with an alkyl azide, the interaction is abolished, explaining the loss in binding. Reinstatement of the Ser hydroxyl group in place of the alkyl azide restored binding affinity $\left(\mathbf{2 2 d}, K_{D}=\right.$ $521-593 \mathrm{nM}$ and $\left.\Delta \mathrm{T}_{\mathrm{m}}=+7.5^{\circ} \mathrm{C}\right)$. Although the binding affinity of $\mathbf{2 2 d}$ fall short of its fully peptidyl counterpart $\left(30, \mathrm{~K}_{\mathrm{D}}=40-84 \mathrm{nM}\right.$ and $\left.\Delta \mathrm{T}_{\mathrm{m}}=+10.4{ }^{\circ} \mathrm{C}\right)$, the permeability of its azide-containing analog 22c was significantly improved relative to 30 (18-hour $\mathrm{EC}_{50}=478$ $\mathrm{nM}$ and 4,106 nM, respectively, Table 3). Considering the historical challenges of finetuning the stability, binding affinity, and permeability, we propose that peptide $\mathbf{2 2 d}$ would provide an attractive template for future discovery aiming to obtain an in vivo tool compound for modulating STUB1. 


\section{Public}

a

\begin{tabular}{|c|c|c|}
\hline Peptide & $\begin{array}{c}\Delta \mathbf{T}_{\mathrm{m}} \\
\left({ }^{\circ} \mathrm{C}, \mathrm{TSA}\right)\end{array}$ & $\begin{array}{c}\mathrm{K}_{\mathrm{D}} \\
(\mathrm{nM}, \mathrm{ITC})\end{array}$ \\
\hline 6 & +13.4 & 30 \\
\hline 28 & +5.8 & $393 ; 764$ \\
\hline 29 & +5.6 & $487 ; 552$ \\
\hline 30 & +10.4 & $40 ; 84$ \\
\hline $22 \mathrm{c}$ & +1.9 & $>10,000$ \\
\hline $22 d$ & +7.5 & $593 ; 521$ \\
\hline
\end{tabular}



d

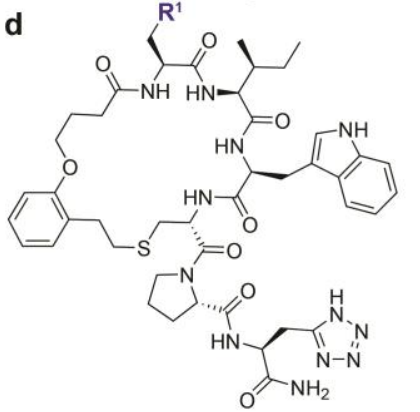

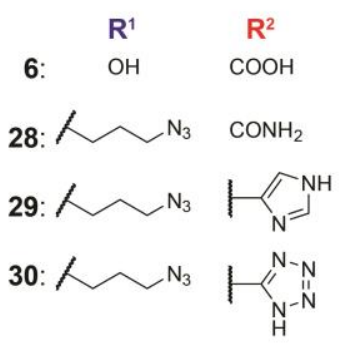

$\mathbf{R}^{1}$

22c: $\sim \mathrm{N}_{3}$

22d: $\mathrm{OH}$

Fig. 4 Mutagenesis of the terminal aspartic acid to fine-tune binding and permeability, and the role of serine at position four. (a) In silico mutation of aspartic acid sidechain ( $\mathrm{n}=$ 9) in peptide 1. (b) Structural variation among peptide 6 and 28-30. (c) Table listing the magnitude of peptide binding to STUB1 as determined by orthogonal biophysical assays. $\Delta T_{m}$ is derived from technical duplicates. $K_{D}$ values from one or two independent experiments were shown explicitly. Complete datasets (TSA and ITC) and repeat experiments are available in supplementary information. (d) Structural variation between peptide 22c and $\mathbf{2 2 d}$.

\section{Conclusions}

Here, multiple classes of mono- and bi-cyclic peptides were identified as potent binders for STUB1 by leveraging $>10^{9}$ peptides displayed on M13 phage. Previous efforts using synthetic combinatorial libraries discovered a closely related linear peptide (LWWPD), ${ }^{18}$ in-line with findings reported here. We observed that macrocyclization of linear peptides with an optimal linkage improved the binding. Building on this, we further optimized the molecules by using phage-discovered macrocyclic peptides and by improving cellular uptake through extensive structure-guided in silico design. The 


\section{Public}

proposed designs successfully replaced a hydrophilic solvent-exposed region with a relatively hydrophobic scaffold, resulting in permeability improvement while maintaining the binding conformation of the macrocyclic peptides. Mutagenesis of the terminal Asp sidechain into tetrazole, a bioisostere of carboxylic acid, partially retained the binding affinity of the peptides while eliminating an anionic residue which is deleterious for cellular uptake. Finally, we uncovered an important role of serine at position four of peptide 1. This finding agrees with the result of phage display - a strong enrichment of either serine or threonine adjacent to an isoleucine in the conserved motifs. We further revealed a key interaction between S4 hydroxyl group of peptide 1 and D134 of STUB1 in the co-crystal structure. It is worth noting that this additional interaction was not found previously using a smaller combinatorial peptide libraries. ${ }^{18}$

Engineering of cell-active peptides is not a trivial task - key attributes, including solubility, stability, permeability, and binding affinity, must be balanced. Each of these attributes is important but becomes limited in isolation. For example, peptides having high binding affinity, but lacking permeability, will not be active in cells, and vice versa. The current lead peptide 22d has reasonable attributes as a starting point $\left(K_{D}=521-593 \mathrm{nM}\right.$, $\Delta \mathrm{T}_{\mathrm{m}}=+7.5^{\circ} \mathrm{C}, t_{1 / 2}=213 \mathrm{~min}, \mathrm{MW}=931 \mathrm{Da}$, and 18-hr. Nanoclick $\mathrm{EC}_{50}=478 \mathrm{nM}$ for 22c), although further improvements will be required before a compound with cellular activity is identified. Given the insights generated in this report and the interest of STUB1 as a potential immuno-oncology target, we anticipate that the current lead peptide will provide a valuable template for the generation of additional tool compounds for intracellular modulation of STUB1 E3 ligase. 


\section{Public}

\section{Conflicts of interest}

There are no conflicts to declare.

\section{Data availability}

Additional information is available in supplementary information which includes supplementary figures, supplementary tables, methods and procedures, complete datasets of TSA, ITC and NanoClick, and characterization of peptides. The list of library oligonucleotides, list of barcoded PCR primers, and coding sequence of M13 phage vector backbone have been uploaded as supplementary files. Crystallographic data for STUB1:peptide 1 complex has been deposited in the Protein Data Bank with accession code [deposition number].

\section{Author Contributions}

Conceptualization: SN, ACB, AWP, ECS, QD; Investigation: SN, ACB, SB, QD, JMJ; Methodology: SN, ACB, SB, LG, RD, B. Habulihaz, BB; Project administration: LG, RD, AS, CY; Software: ACB, QD, JMJ, SH; Supervision: AWP, ECS, B. Henry, AP, CS, QD; Visualization: SN, ACB; Writing - original draft: SN, ACB; Writing - review \& editing: AWP, ECS, QD, JMJ.

\section{Acknowledgements}

We are grateful for the financial support from MSD. SN and ACB acknowledge support from the MRL Postdoctoral Research Program. We thank Christian Fischer for feedback and proofreading of the manuscript. We are grateful for Daniel J. Klein's contribution in 


\section{Public}

the methodology and deposition of crystallographic data. The protein production effort of

NTU PPP is appreciated. We further thank Chinese Peptide Company and WuXi AppTec

for the peptide synthesis support.

\section{References}

(1) Connell, P.; Ballinger, C. A.; Jiang, J.; Wu, Y.; Thompson, L. J.; Höhfeld, J.; Patterson, $\mathrm{C}$. The co-chaperone CHIP regulates protein triage decisions mediated by heat-shock proteins. Nat. Cell Biol. 2001, 3, 93-96.

(2) McDonough, H.; Patterson, C. CHIP: a link between the chaperone and proteasome systems. Cell Stress Chaperones 2003, 8, 303-308.

(3) Kundrat, L.; Regan, L. Balance between Folding and Degradation for Hsp90Dependent Client Proteins: A Key Role for CHIP. Biochemistry 2010, 49, 7428-7438.

(4) Joshi, V.; Amanullah, A.; Upadhyay, A.; Mishra, R.; Kumar, A.; Mishra, A. A Decade of Boon or Burden: What Has the CHIP Ever Done for Cellular Protein Quality Control Mechanism Implicated in Neurodegeneration and Aging? Front. Mol. Neurosci. 2016, 9.

(5) Kumar, S.; Basu, M.; Ghosh, M. K. Chaperone-assisted E3 ligase CHIP: A double agent in cancer. Genes \& Diseases 2021.

(6) Morishima, Y.; Wang, A. M.; Yu, Z.; Pratt, W. B.; Osawa, Y.; Lieberman, A. P. CHIP deletion reveals functional redundancy of $\mathrm{E} 3$ ligases in promoting degradation of both signaling proteins and expanded glutamine proteins. Hum. Mol. Genet. 2008, 17, 39423952.

(7) Min, J.-N.; Whaley Ryan, A.; Sharpless Norman, E.; Lockyer, P.; Portbury Andrea, L.; Patterson, C. CHIP Deficiency Decreases Longevity, with Accelerated Aging Phenotypes Accompanied by Altered Protein Quality Control. Mol. Cell. Biol. 2008, 28, 4018-4025.

(8) Dai, Q.; Zhang, C.; Wu, Y.; McDonough, H.; Whaley, R. A.; Godfrey, V.; Li, H.-H.; Madamanchi, N.; Xu, W.; Neckers, L.; Cyr, D.; Patterson, C. CHIP activates HSF1 and confers protection against apoptosis and cellular stress. The EMBO Journal 2003, 22, $5446-5458$.

(9) Tawo, R.; Pokrzywa, W.; Kevei, É.; Akyuz, M. E.; Balaji, V.; Adrian, S.; Höhfeld, J.; Hoppe, T. The Ubiquitin Ligase CHIP Integrates Proteostasis and Aging by Regulation of Insulin Receptor Turnover. Cell 2017, 169, 470-482.e13.

(10) Heimdal, K.; Sanchez-Guixé, M.; Aukrust, I.; Bollerslev, J.; Bruland, O.; Jablonski, G. E.; Erichsen, A. K.; Gude, E.; Koht, J. A.; Erdal, S.; Fiskerstrand, T.; Haukanes, B. I.; Boman, H.; Bjørkhaug, L.; Tallaksen, C. M. E.; Knappskog, P. M.; Johansson, S. STUB1 mutations in autosomal recessive ataxias - evidence for mutation-specific clinical heterogeneity. Orphanet J. Rare Dis. 2014, 9, 146.

(11) Chen, Z.; Barbi, J.; Bu, S.; Yang, H.-Y.; Li, Z.; Gao, Y.; Jinasena, D.; Fu, J.; Lin, F.; Chen, C.; Zhang, J.; Yu, N.; Li, X.; Shan, Z.; Nie, J.; Gao, Z.; Tian, H.; Li, Y.; Yao, Z.; Zheng, Y.; Park, Benjamin V.; Pan, Z.; Zhang, J.; Dang, E.; Li, Z.; Wang, H.; Luo, W.; Li, L.; Semenza, Gregg L.; Zheng, S.-G.; Loser, K.; Tsun, A.; Greene, Mark I.; Pardoll, Drew M.; Pan, F.; Li, B. The Ubiquitin Ligase Stub1 Negatively Modulates Regulatory T 


\section{Public}

Cell Suppressive Activity by Promoting Degradation of the Transcription Factor Foxp3. Immunity 2013, 39, 272-285.

(12) Zhou, P.; Ding, X.; Wan, X.; Liu, L.; Yuan, X.; Zhang, W.; Hui, X.; Meng, G.; Xiao, H.; Li, B.; Zhong, J.; Hou, F.; Deng, L.; Zhang, Y. MLL5 suppresses antiviral innate immune response by facilitating STUB1-mediated RIG-I degradation. Nat. Commun. 2018, 9, 1243.

(13) Manguso, R. T.; Pope, H. W.; Zimmer, M. D.; Brown, F. D.; Yates, K. B.; Miller, B. C.; Collins, N. B.; Bi, K.; LaFleur, M. W.; Juneja, V. R.; Weiss, S. A.; Lo, J.; Fisher, D. E.; Miao, D.; Van Allen, E.; Root, D. E.; Sharpe, A. H.; Doench, J. G.; Haining, W. N. In vivo CRISPR screening identifies Ptpn2 as a cancer immunotherapy target. Nature 2017, 547, 413-418.

(14) Mezzadra, R.; Sun, C.; Jae, L. T.; Gomez-Eerland, R.; de Vries, E.; Wu, W.; Logtenberg, M. E. W.; Slagter, M.; Rozeman, E. A.; Hofland, I.; Broeks, A.; Horlings, H. M.; Wessels, L. F. A.; Blank, C. U.; Xiao, Y.; Heck, A. J. R.; Borst, J.; Brummelkamp, T. R.; Schumacher, T. N. M. Identification of CMTM6 and CMTM4 as PD-L1 protein regulators. Nature 2017, 549, 106-110.

(15) Rebeyev, N. CHIC2 and STUB1 regulate interferon-y receptor cell surface expression. University of Cambridge (Doctoral thesis), 2020.

(16) Apriamashvili, G.; Vredevoogd, D. W.; Krijgsman, O.; Bleijerveld, O. B.; Ligtenberg, M. A.; de Bruijn, B.; Boshuizen, J.; Altimari, D. D. E.; Visser, N. L.; Londino, J. D.; Altelaar, M.; Peeper, D. S. Loss of Ubiquitin Ligase STUB1 Amplifies IFNy-R1/JAK1 Signaling and Sensitizes Tumors to IFNy. bioRxiv 2020, 2020.07.07.191650.

(17) Ng, S.; Lim, S.; Sim, A. C. N.; Mangadu, R.; Lau, A.; Zhang, C.; Martinez, S. B.; Chandramohan, A.; Lim, U. M.; Ho, S. S. W.; Chang, S. C.; Gopal, P.; Hong, L. Z.; Schwaid, A.; Fernandis, A. Z.; Loboda, A.; Li, C.; Phan, U.; Henry, B.; Partridge, A. W. STUB1 is an intracellular checkpoint for interferon gamma sensing. bioRxiv 2021, 2020.12.14.420539.

(18) Ravalin, M.; Theofilas, P.; Basu, K.; Opoku-Nsiah, K. A.; Assimon, V. A.; MedinaCleghorn, D.; Chen, Y.-F.; Bohn, M. F.; Arkin, M.; Grinberg, L. T.; Craik, C. S.; Gestwicki, J. E. Specificity for latent $\mathrm{C}$ termini links the E3 ubiquitin ligase CHIP to caspases. Nat. Chem. Biol. 2019, 15, 786-794.

(19) Marsault, E.; Peterson, M. L. Macrocycles Are Great Cycles: Applications, Opportunities, and Challenges of Synthetic Macrocycles in Drug Discovery. J. Med. Chem. 2011, 54, 1961-2004.

(20) Nadel, C. M.; Ran, X.; Gestwicki, J. E. Luminescence complementation assay for measurement of binding to protein C-termini in live cells. Anal. Biochem. 2020, 611, 113947.

(21) Basheer, M.; Schwalb, H.; Shefler, I.; Levdansky, L.; Mekori, Y. A.; Gorodetsky, R. Blood pressure modulation following activation of mast cells by cationic cell penetrating peptides. Peptides 2011, 32, 2444-2451.

(22) Lu, L.; Kulka, M.; Unsworth, L. D. Peptide-mediated mast cell activation: ligand similarities for receptor recognition and protease-induced regulation. J. Leukoc. Biol. 2017, 102, 237-251.

(23) Lim, S.; Boyer, N.; Boo, N.; Huang, C.; Venkatachalam, G.; Angela Juang, Y.-C.; Garrigou, M.; Kaan, H. Y. K.; Duggal, R.; Peh, K. M.; Sadruddin, A.; Gopal, P.; Yuen, T. Y.; Ng, S.; Kannan, S.; Brown, C. J.; Verma, C. S.; Orth, P.; Peier, A.; Ge, L.; Yu, X.; 


\section{Public}

Bhatt, B.; Chen, F.; Wang, E.; Li, N. J.; Gonzales, R. J.; Stoeck, A.; Henry, B.; Sawyer, T. K.; Lane, D. P.; Johannes, C. W.; Biswas, K.; Partridge, A. W. Discovery of cell active macrocyclic peptides with on-target inhibition of KRAS signaling. Chemical Science 2021. (24) Craik, D. J.; Fairlie, D. P.; Liras, S.; Price, D. The Future of Peptide-based Drugs. Chem. Biol. Drug Des. 2013, 81, 136-147.

(25) Wells, J. A.; McClendon, C. L. Reaching for high-hanging fruit in drug discovery at protein-protein interfaces. Nature 2007, 450, 1001-1009.

(26) Vinogradov, A. A.; Yin, Y.; Suga, H. Macrocyclic Peptides as Drug Candidates: Recent Progress and Remaining Challenges. J. Am. Chem. Soc. 2019, 141, 4167-4181. (27) Tucker, T. J.; Embrey, M. W.; Alleyne, C.; Amin, R. P.; Bass, A.; Bhatt, B.; Bianchi, E.; Branca, D.; Bueters, T.; Buist, N.; Ha, S. N.; Hafey, M.; He, H.; Higgins, J.; Johns, D. G.; Kerekes, A. D.; Koeplinger, K. A.; Kuethe, J. T.; Li, N.; Murphy, B.; Orth, P.; Salowe, S.; Shahripour, A.; Tracy, R.; Wang, W.; Wu, C.; Xiong, Y.; Zokian, H. J.; Wood, H. B.; Walji, A. A Series of Novel, Highly Potent, and Orally Bioavailable Next-Generation Tricyclic Peptide PCSK9 Inhibitors. J. Med. Chem. 2021.

(28) Kale, S. S.; Villequey, C.; Kong, X.-D.; Zorzi, A.; Deyle, K.; Heinis, C. Cyclization of peptides with two chemical bridges affords large scaffold diversities. Nat. Chem. 2018, 10, 715-723.

(29) Ng, S.; Derda, R. Phage-displayed macrocyclic glycopeptide libraries. Org. Biomol. Chem. 2016, 14, 5539-5545.

(30) Iskandar, S. E.; Haberman, V. A.; Bowers, A. A. Expanding the Chemical Diversity of Genetically Encoded Libraries. ACS Combinatorial Science 2020, 22, 712-733.

(31) Ng, S.; Lin, E.; Kitov, P. I.; Tjhung, K. F.; Gerlits, O. O.; Deng, L.; Kasper, B.; Sood, A.; Paschal, B. M.; Zhang, P.; Ling, C.-C.; Klassen, J. S.; Noren, C. J.; Mahal, L. K.; Woods, R. J.; Coates, L.; Derda, R. Genetically Encoded Fragment-Based Discovery of Glycopeptide Ligands for Carbohydrate-Binding Proteins. J. Am. Chem. Soc. 2015, 137, 5248-5251.

(32) Ng, S.; Bennett, N. J.; Schulze, J.; Gao, N.; Rademacher, C.; Derda, R. Geneticallyencoded fragment-based discovery of glycopeptide ligands for DC-SIGN. Biorg. Med. Chem. 2018, 26, 5368-5377.

(33) Tjhung, K. F.; Kitov, P. I.; Ng, S.; Kitova, E. N.; Deng, L.; Klassen, J. S.; Derda, R. Silent Encoding of Chemical Post-Translational Modifications in Phage-Displayed Libraries. J. Am. Chem. Soc. 2016, 138, 32-35.

(34) Ng, S.; Juang, Y.-C.; Chandramohan, A.; Kaan, H. Y. K.; Sadruddin, A.; Yuen, T. Y.; Ferrer-Gago, F. J.; Lee, X. E. C.; Liew, X.; Johannes, C. W.; Brown, C. J.; Kannan, S.; Aronica, P. G.; Berglund, N. A.; Verma, C. S.; Liu, L.; Stoeck, A.; Sawyer, T. K.; Partridge, A. W.; Lane, D. P. De-risking Drug Discovery of Intracellular Targeting Peptides: Screening Strategies to Eliminate False-Positive Hits. ACS Med. Chem. Lett. 2020, 11, 1993-2001.

(35) Walport, L. J.; Low, J. K. K.; Matthews, J. M.; Mackay, J. P. The characterization of protein interactions - what, how and how much? Chem. Soc. Rev. 2021.

(36) Matsson, P.; Kihlberg, J. How Big Is Too Big for Cell Permeability? J. Med. Chem. 2017, 60, 1662-1664.

(37) Pye, C. R.; Hewitt, W. M.; Schwochert, J.; Haddad, T. D.; Townsend, C. E.; Etienne, L.; Lao, Y.; Limberakis, C.; Furukawa, A.; Mathiowetz, A. M.; Price, D. A.; Liras, S.; Lokey, 


\section{Public}

R. S. Nonclassical Size Dependence of Permeation Defines Bounds for Passive Adsorption of Large Drug Molecules. J. Med. Chem. 2017, 60, 1665-1672.

(38) Zhang, M.; Windheim, M.; Roe, S. M.; Peggie, M.; Cohen, P.; Prodromou, C.; Pearl, L. H. Chaperoned Ubiquitylation-Crystal Structures of the CHIP U Box E3 Ubiquitin Ligase and a CHIP-Ubc13-Uev1a Complex. Mol. Cell 2005, 20, 525-538.

(39) Wang, L.; Liu, Y.-T.; Hao, R.; Chen, L.; Chang, Z.; Wang, H.-R.; Wang, Z.-X.; Wu, J.-W. Molecular Mechanism of the Negative Regulation of Smad1/5 Protein by Carboxyl Terminus of Hsc70-interacting Protein (CHIP). J. Biol. Chem. 2011, 286, 15883-15894.

(40) Peier, A.; Ge, L.; Boyer, N.; Frost, J.; Duggal, R.; Biswas, K.; Edmondson, S.; Hermes, J. D.; Yan, L.; Zimprich, C.; Sadruddin, A.; Kristal Kaan, H. Y.; Chandramohan, A.; Brown, C. J.; Thean, D.; Lee, X. E.; Yuen, T. Y.; Ferrer-Gago, F. J.; Johannes, C. W.; Lane, D. P.; Sherborne, B.; Corona, C.; Robers, M. B.; Sawyer, T. K.; Partridge, A. W. NanoClick: A High Throughput, Target-Agnostic Peptide Cell Permeability Assay. ACS Chem. Biol. 2021, 16, 293-309.

(41) Kale, S. S.; Bergeron-Brlek, M.; Wu, Y.; Kumar, M. G.; Pham, M. V.; Bortoli, J.; Vesin, J.; Kong, X. D.; Machado, J. F.; Deyle, K.; Gonschorek, P.; Turcatti, G.; Cendron, L.; Angelini, A.; Heinis, C. Thiol-to-amine cyclization reaction enables screening of large libraries of macrocyclic compounds and the generation of sub-kilodalton ligands. Science Advances 2019, 5, eaaw2851.

(42) Jwad, R.; Weissberger, D.; Hunter, L. Strategies for Fine-Tuning the Conformations of Cyclic Peptides. Chem. Rev. 2020, 120, 9743-9789.

(43) Wang, L.; Friesner, R. A.; Berne, B. J. Replica Exchange with Solute Scaling: A More Efficient Version of Replica Exchange with Solute Tempering (REST2). The Journal of Physical Chemistry B 2011, 115, 9431-9438.

(44) Diamond, J. M.; Katz, Y. Interpretation of nonelectrolyte partition coefficients between dimyristoyl lecithin and water. The Journal of Membrane Biology 1974, 17, 121154.

(45) Walter, A.; Gutknecht, J. Permeability of small nonelectrolytes through lipid bilayer membranes. The Journal of Membrane Biology 1986, 90, 207-217.

(46) Molecular Operating Environment (MOE); Chemical Computing Group ULC, 1010 Sherbooke St. West, Suite \#910, Montreal, QC, Canada, H3A 2 R7.

(47) Partridge, A. W.; Kaan, H. Y.; Juang, Y.-C.; Sadruddin, A.; Lim, S.; Brown, C. J.; Ng, S.; Thean, D.; Ferrer, F.; Johannes, C.; Yuen, T. Y.; Kannan, S.; Aronica, P.; Tan, Y. S.; Pradhan, M. R.; Verma, C. S.; Hochman, J.; Chen, S.; Wan, H.; Ha, S.; Sherborne, B.; Lane, D. P.; Sawyer, T. K. Incorporation of Putative Helix-Breaking Amino Acids in the Design of Novel Stapled Peptides: Exploring Biophysical and Cellular Permeability Properties. Molecules 2019, 24.

(48) Kannan, S.; Aronica, P. G. A.; Ng, S.; Gek Lian, D. T.; Frosi, Y.; Chee, S.; Shimin, J.; Yuen, T. Y.; Sadruddin, A.; Kaan, H. Y. K.; Chandramohan, A.; Wong, J. H.; Tan, Y. S.; Chang, Z. W.; Ferrer-Gago, F. J.; Arumugam, P.; Han, Y.; Chen, S.; Rénia, L.; Brown, C. J.; Johannes, C. W.; Henry, B.; Lane, D. P.; Sawyer, T. K.; Verma, C. S.; Partridge, A. W. Macrocyclization of an all-d linear a-helical peptide imparts cellular permeability. Chemical Science 2020, 11, 5577-5591.

(49) Yang, N. J.; Hinner, M. J., Getting Across the Cell Membrane: An Overview for Small Molecules, Peptides, and Proteins. In Site-Specific Protein Labeling: Methods and 


\section{Public}

Protocols, Gautier, A.; Hinner, M. J., Eds. Springer New York: New York, NY, 2015; pp 29-53. 